\title{
Stretchable capacitive tactile skin on humanoid robot fingers - first experiments and results
}

\author{
Aaron P. Gerratt ${ }^{1}$, Nicolas Sommer ${ }^{2}$, Stéphanie P. Lacour ${ }^{1}$, and Aude Billard ${ }^{2}$
}

\begin{abstract}
A stretchable tactile sensor skin has been demonstrated on the dorsal side of a robotic hand for the first time. The sensors can detect normal pressures on the same scale as human skin but also in excess of $250 \mathrm{kPa}$ and withstand strains in excess of $15 \%$. Using tactile information from the sensors mounted on a glove worn by a humanoid robot's hand, obstacle detection and surface reconstruction tasks were successfully completed in order to demonstrate the performance of the sensors under applied strains and pressure.
\end{abstract}

\section{INTRODUCTION}

Recent advances in mechanisms for complex manipulation and grasping have created a demand for compatible sensors for external stimuli such as pressure. An ideal solution is an electronic skin that is mechanically similar to and has similar embedded sensory capabilities as human skin. Biological pressure transducers can differentiate shear and normal pressures from a light touch of just 100s of Pa to heavy loads on the order of $1 \mathrm{MPa}$, and can do so while remaining mechanically compliant [1]. While there are examples of rigid systems with sensory capabilities that can detect pressure across similar dynamic ranges, the remaining challenge is in the integration of sensors that mimic both the sensory capabilities and mechanical properties of skin, particularly in terms of mechanical stretchability.

There are many examples of sensors that have been manufactured using soft materials on top of a rigid substrate. These sensors have led to papers demonstrating remarkable pressure sensitivities, some exceeding that of human skin, but the use of rigid materials in the construction makes them difficult to integrate on mobile robotic systems [2], [3], [4]. Accordingly, these sensors are usually implemented as patches placed on top of a hand or along an arm in locations that are flat or have

\footnotetext{
${ }^{1}$ Laboratory for Soft Bioelectronic Interfaces, École Polytechnique Fédérale de Lausanne, Lausanne, Switzerland stephanie.lacour@epfl.ch

${ }^{2}$ Learning Algorithms and Systems Laboratory, École Polytechnique Fédérale de Lausanne, Lausanne, Switzerland aude.billardeepfl.ch
}

a large radius of curvature and where complex motions do not occur. These solutions are not suitable for use in areas of more intricate motion, particularly joints.

Sensors must be able to accommodate simultaneous multi-directional deformations in at least two directions at joints: around the body and orthogonally in the direction of the joint bending. This requirement is exacerbated at knuckles on fingers where the radii of curvatures in both directions are small, on the order of single millimeters. The two degrees of freedom require fully stretchable materials in order to avoid buckling when bending.

The two most common sensing modes for flexible and stretchable sensors are resistive and capacitive, though transduction mechanisms such as optical [5] and piezoelectric [6] have also been demonstrated. Stretchable resistive sensors are often prepared with conductive composites using metallic particles, graphene or carbon nanotubes in an elastomer matrix [7], and more recently with liquid metal [8], [9]. Resistive sensors are convenient because of the their ease of fabrication and integration with electronic hardware. However they are characterized by small dynamic ranges, large hysteresis, and low sensitivity [8], [9], [10]. Capacitive sensors require more complex fabrication and read-out electronics, but have been demonstrated with higher fidelity outputs and larger dynamic ranges.

Several designs for stretchable tactile skins are being explored and their complete integration in a wearable system remains a technological challenge [8], [11], [12], [13]. One example of a stretchable skin with embedded pressure sensing uses carefully engineered meanders of a rigid polyimide/metal multilayer structure to address individual nodes [11]. The electrodes at the nodes are made of this rigid multilayer structure, so the sensor nodes themselves are not stretchable. One of the best examples of arrayed stretchable tactile sensors implemented in a robotic or wearable application uses layered conductive fabrics stacked and sewn together as a glove [12]. The 54 pressure-dependent resistive nodes in the device can be addressed in real time. 
Tactile sensors have already been used for haptic exploration in our previous works, by providing compliance in the finger motion for recognizing humanlike faces by touch [14], and for 3D reconstruction and identification of objects in the context of bimanual exploration [15]. Sensors were only using the palmar side of the hand, however.

Demonstrations and implementations of tactile sensors are primarily along the palmar side of fingers and hands, motivated by grasping and manipulation tasks. The palmar side of the finger primarily experiences compression, which is less demanding than tensile deformation. Sensors on the outside, or dorsal side, of the finger are useful for environmental exploration in settings where the hand is closed, for contact detection when already grasping an object, or for exploration of the inside of an object. Current sensors cannot be positioned at the bendable joints of the finger. Contacts, however, tend to occur more often at the edges (for instance, knuckles and elbow). For this reason, having stretchable sensors at that position is crucial for controlling a robot in contact with its environment. The goal of this paper is to review our soft sensor technology and demonstrate their potential in tactile exploration tasks. This is the first demonstration of a stretchable tactile sensor skin mounted on the dorsal side of a robotic finger.

\section{SENSOR DESIGN AND FABRICATION}

The goal of stretchable sensors dictates that elastomeric materials be used for the structural layers. One of the drawbacks of bulk elastomers, however, is that they are incompressible (Poisson's ratio of about 0.5 ). This is a limiting factor for capacitive sensors, as the output of the sensor is proportional to the compression of the dielectric material. The capacitance per unit area, $C / A$, of a parallel-plate sensor can be calculated as

$$
C / A=\frac{\epsilon_{0} \epsilon_{r}}{t}
$$

where $\epsilon_{0}$ is the vacuum permittivity, $\epsilon_{r}$ is the dielectric constant of the material separating the two electrode, and $t$ is the gap separating the electrodes.

Bulk silicone elastomers commonly used in stretchable electronics including poly(dimethylsiloxane) (PDMS) and Ecoflex are incompressible and have non-linear elastic moduli that increase with increased applied tensile and compressive strains. They are thus not ideal for soft compression sensors.

The sensor dynamic range and sensitivity can be improved using a porous elastomer, i.e. polyurethane foam [16] instead of a plain, bulk membrane. Here we introduce the use of a silicone foam as a highly sensitive dielectric membrane. Stretchable electrodes prepared as a bi-layer of $5 \mathrm{~nm} \mathrm{Cr}$ and $30 \mathrm{~nm} \mathrm{Au}$ films are evaporated and patterned on a thin PDMS membrane which is subsequently bonded (irreversibly) with the silicone foam. The stretchability of the thin films of gold on PDMS silicone substrates has been fully characterized in previous publications [17]. In brief, the bi-layer films have a randomly oriented microcracks distributed throughout the surface. Under mechanical elongation, strain relief is provided by these microcracks which open and bend out-of-plane, rather than through cracking, as would occur with a thicker, bulk electrode.

Silicone substrates for the electrodes are prepared by spin coating prior to polymerization, which allows for dictating the layer thickness by adjusting the speed of rotation. The electrodes are patterned on top of the silicone substrates by thermal deposition through shadow masks. This fabrication allows for batch as well as large-area substrate processing. The multiple layers are prepared separately and then bonded together with a brief exposure to an oxygen plasma just prior to being placed in contact, resulting in irreversible covalent bonding. The electrical traces in the multilayer structure are connected together with a conductive silver particle composite.

The fabricated devices have four $\mathrm{Cr} / \mathrm{Au}$ bi-layers. A cross-section schematic of a device is shown in Fig. 1a which shows two layers that form the parallel plate sensors between which the capacitance is measured as well as the shielding and ground layers. In total, six $50 \mu \mathrm{m}$ thick layer of silicone and one $880 \mu \mathrm{m}$ thick layer of foam are bonded together to form the multilayer structure with a total thickness of $1.18 \mathrm{~mm}$. The aggregate thickness of the four electrode layers is only $140 \mathrm{~nm}$. The six sensor nodes shown in Fig. 1b are $5 \mathrm{~mm}$ wide and $9 \mathrm{~mm}$ long with a $1 \mathrm{~mm}$ spacing.

The final step in the fabrication is the connection of a printed circuit board with a capacitance-to-digital converter (CDC) to the electrodes with the silver composite, as shown in Fig. 1b. The silver composite creates a robust compliant electrical connection between the rigid PCB and the soft, stretchable gold electrodes. The composite remains a viscous fluid, but is encapsulated in a room temperature vulcanising silicone, providing a compliant link. The 16-bit CDC, an AD7147 from Analog Devices, provides an AC shield signal that mimics the signal applied to the electrodes in order to 


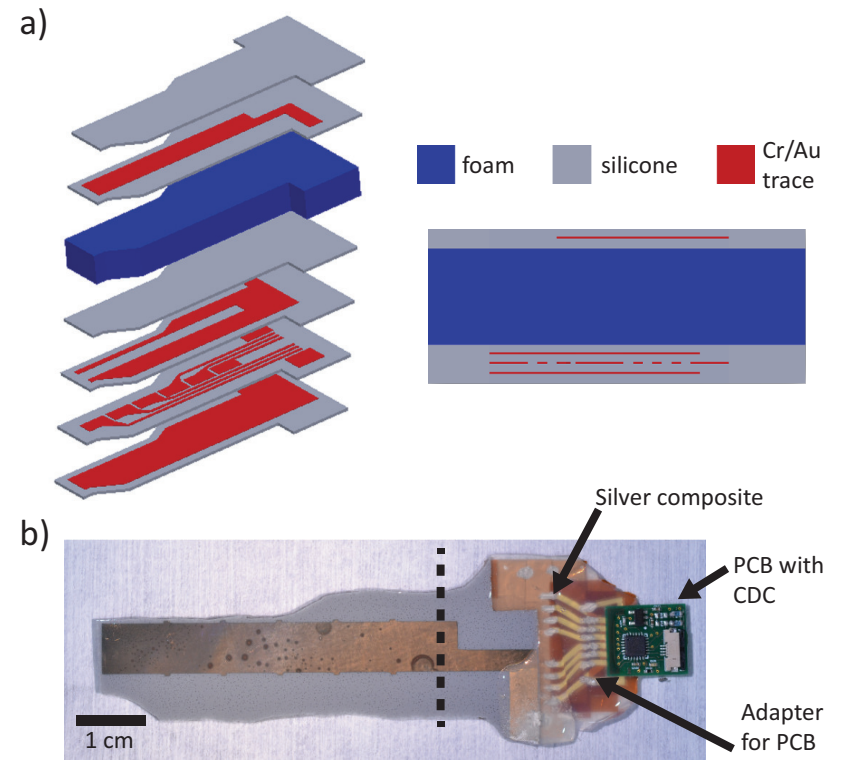

Fig. 1: a) An exploded (left) and cross-section (right) schematic view of a stretchable tactile skin designed to coat a finger. The electrode layers, top-down, are the ground, top shield layer, electrode and trace layer, and the bottom shield layer (not to scale). b) A fabricated sensor prior to being mounted on a textile glove. The dashed line indicates the location of the cross-section shown above.

isolate the sensor electrode and traces from any external electric fields and limit noise and prevents node-to-node cross-sensitivity. These are important requirements for robotics applications as the sensors are likely to be placed directly above other electronics and motors. This is also important for wearable applications where proximity to the body can introduce significant noise to unshielded capacitive sensors.

\section{SENSOR CHARACTERIZATION}

A simplified version of the capacitors shown in Figure 1 was used to characterize the performance of the sensors under applied pressures and strains. This single node device consisted of two $1 \mathrm{~cm}^{2}$ electrodes on either side of a foam membrane. Compressive loads were applied with a C42 universal testing system from MTS with a $100 \mathrm{~N}$ load with an accuracy of $\pm 0.5 \mathrm{~N}$, which corresponds to $\pm 2.6 \mathrm{kPa}$ in the test setup. A test of the response to pressure was performed by stepping the applied pressure to $260 \mathrm{kPa}$. In order to quantify the change in the response of the sensors due to an applied axial strain, the pressure sensitivity test was performed at $0 \%$ and $15 \%$ applied strain, which was controlled by

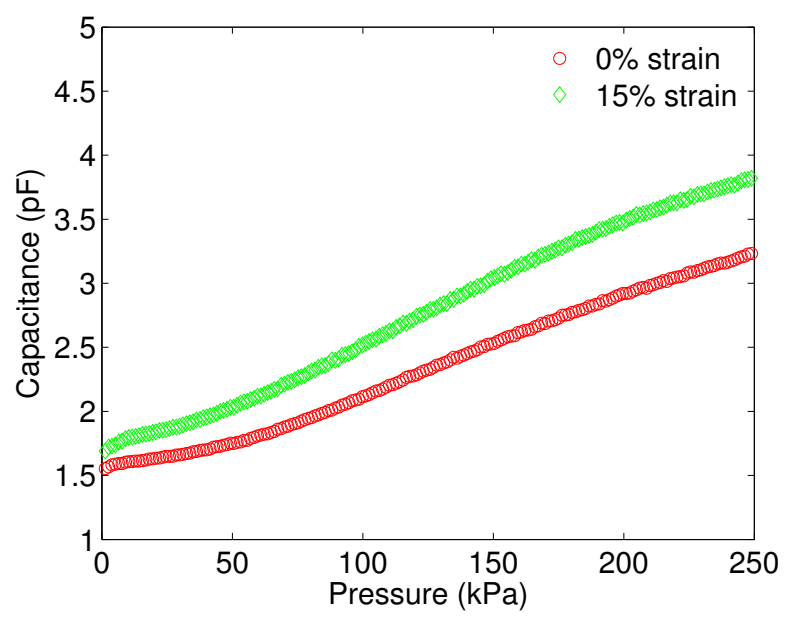

Fig. 2: Change in capacitance as a function of applied stress and strain.

a custom manual uniaxial stage. The capacitance was measured with an Agilent E4980A LCR meter.

The response of the sensor to the applied stress and strain, shown in Figure 2, indicates that a measure of the strain is necessary in order to decouple the response of the sensors to the combined pressure and strain. In a robotics context, this decoupling can be achieved by utilizing the existing infrastructure in robots. This work uses joint angle sensors at each of the fingers of the iCub humanoid robot, which will be discussed in Section IV, to aid in decoupling the contributions of strain and pressure to the sensor output. The joint sensors are used to detect movement of the fingers. When the fingers are not moving and it is known that contact is not occurring, we zero the sensor's response by removing the sensor offset corresponding to the current finger position and thus sensor bending, i.e. the sensor values being read. This is sufficient for preliminary proof-of-concept work for elimination of false-positive detections of contact at light pressures. There are, however, many examples of stretchable strain sensors which can be used to provide complete proprioceptive information, and integration of stretchable strain and pressure sensors remains a topic of future work for more generalized implementation of the sensors in scenarios without joint sensors.

Figure 3 illustrates the sensing range of the sensors. The input pressure was applied in incremental steps ranging for $5 \mathrm{kPa}$ to $230 \mathrm{kPa}$. Three sequences are shown Figure 3. The three-step pressure cycle was first $5.2-13-26 \mathrm{kPa}$, then $26-52-77 \mathrm{kPa}$, and finally 77 $155-232 \mathrm{kPa}$. Each cycle was repeated 5 times before 


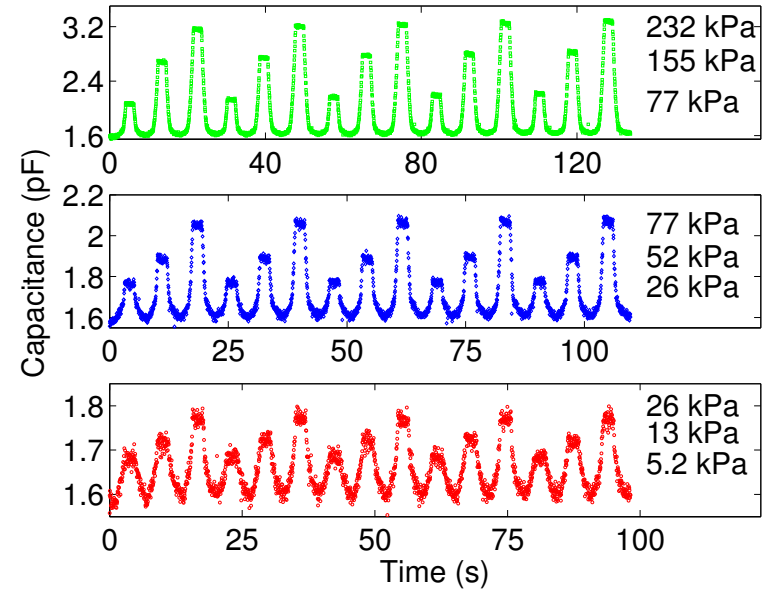

Fig. 3: Stepped pressure in three pressure ranges with five cycles per range.

moving on to the next pressure level. The pressure was applied with an indenter moving at a constant rate of $0.5 \mathrm{~mm} / \mathrm{s}$. The time scales on the three plots vary slightly due to the difference in the total displacement of the indenter during the application and removal of the pressure. The results shown in Figure 3 demonstrate the discrimination of pressures from 5 to $232 \mathrm{kPa}$, though the full range extends above and below this range, and shows little hysteresis. This pressure range is comparable to that of human skin. The task of typing requires approximately $5 \mathrm{kPa}$ [18] while grasping tasks require pressures on the order of $10 \mathrm{kPa}$ [19]. More generally, the sensory requirements for tactile skin range from 1 to $1000 \mathrm{kPa}$ [1]. The implementation of these sensors for tactile exploration utilizing the pressure response of the sensors is demonstrated in the following section. The experiments demonstrated in Section IV use the sensors at the lower end of their dynamic range, for tasks that require sensing pressures up to $10 \mathrm{kPa}$.

Next we evaluated the sensor response to prolonged compressive loading. Figure 4a shows the response to a compressive pressure of $103 \mathrm{kPa}$. The sensor was first kept at rest for 10 minutes, compressed for 10 minutes, and then kept at rest for another 10 minutes. During the 10 minutes of applied pressure we observed a small increase in the measured capacitance, most likely due to both the relaxation of the polymer under compression and migration of the air through the opencell network of the foam. During a similar test, a sample was kept at rest for 10 minutes, elongated to $15 \%$ strain and held for 10 minutes, and then returned a)

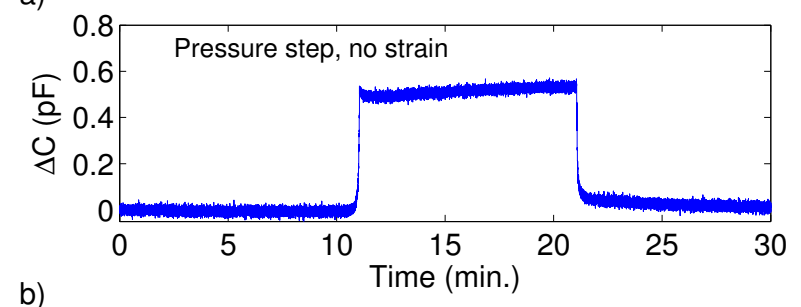

b)

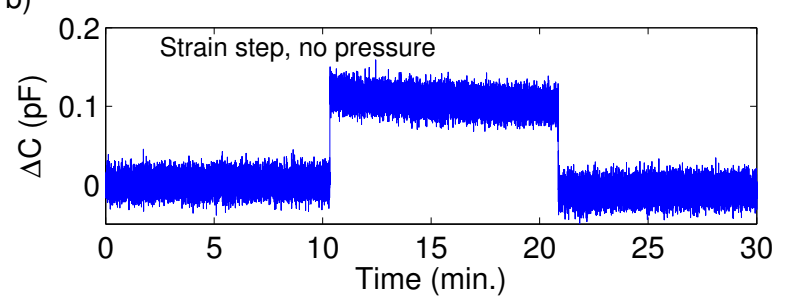

Fig. 4: a) A step to a compressive pressure of $103 \mathrm{kPa}$ held for 10 minutes. b) A step to a tensile strain of $15 \%$ held for 10 minutes.

to $0 \%$ strain and monitored for another 10 minutes, shown in Figure 4b. The recorded noise level appears artificially larger than in the previous experiment, as the scale of the capacitance change is not the same. During prolonged stretching, the recorded capacitance is stable and recovers its initial value upon release of the strain. Next we examined the stability of the soft pressure sensor with cyclic pressure loading. 130 compression cycles to $300 \mathrm{kPa}$ pressure were applied to the sensor surface with an indenter moving at constant compression rate of $0.1 \mathrm{~mm} / \mathrm{s}$. The sensor is robust and its response is stable during the cyclic loading. There was a small increase in the baseline, zero pressure capacitance, as well as a small increase in the maximum capacitance measure with the applied pressure, shown in Figure 5a with the first and last 5 cycles shown in Figures $5 b$ and $5 c$, respectively. These increases are likely due to the same relaxation noticed in Figure 4a.

\section{ROBOT INTEGRATION}

The motivation for the development of the sensors characterized in Section III is to demonstrate transduction of applied pressures on the dorsal side of the finger. Section III focused on characterization in a laboratory setting, so in order to demonstrate their efficacy in a more generalized environment, sensors are mounted on a stretchable textile glove and fitted onto the hand of the iCub humanoid robot. The sensors are manufactured in sets of six $9 \mathrm{~mm} \times 5 \mathrm{~mm}$ nodes distributed along the length of the finger, as shown in Figure 1b. The sensor acquisition rate during these tests was approximately 
a)

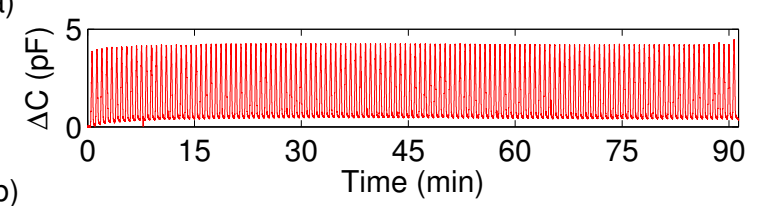

b)

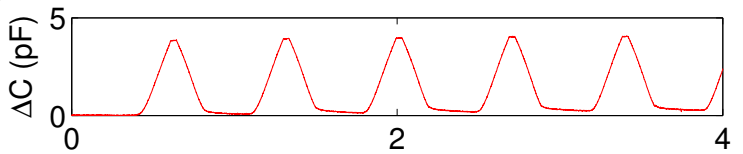

c)

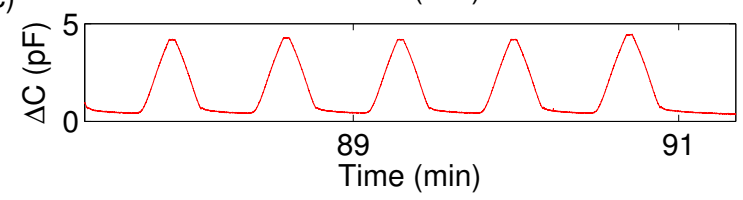

Fig. 5: a) 130 cycles to $300 \mathrm{kPa}$. b) The first four minutes of the test. c) The last 4 minutes of the tes.

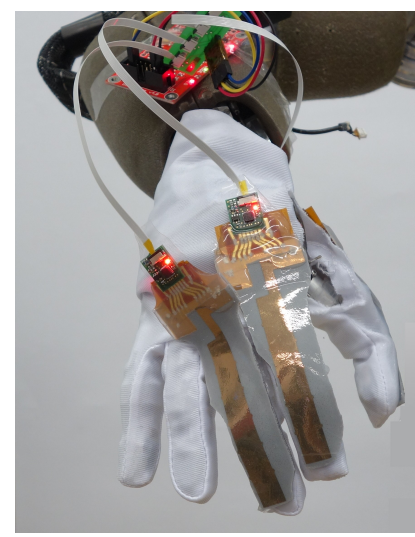

(a)

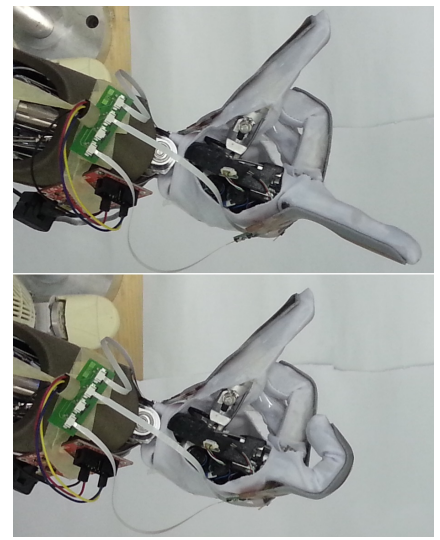

(b)
Fig. 6: a) The hand of the iCub humanoid robot with tactile sensors mounted on the back of its fingers. b) The sensors stretch and bend with the fingers.

$20 \mathrm{~Hz}$, though this can be increased in future work by improving the serial communication. The $\mathrm{iCub}$ is used for two different applications of the sensors described above. In both experiments, we are using the tactile skin to detect contact on the back of the fingers and to provide compliance in the finger motion. In the first experiment, the sensors are used to detect contact with an obstacle during the arm's motion. In the second experiment, the fingers make use of the sensor pressure information to compliantly explore haptic features.

\section{A. Experiments}

1) Setup: The iCub Humanoid robot is a 53-DOFs humanoid robot whose arms are composed of 7 joints, plus 9-DOFs hands. The 7 arm joints are used to achieve the motion of the hand while one joint per finger is used to follow the surface in the second experiment (index, middle and thumb fingers can be used for this experiment ${ }^{1}$ ). The tactile sensors are mounted on the back of the fingers: each finger is equipped with 6 tactile patches uniformly distributed from the first phalanx until the fingertip (see Figure 6).

Each finger has 3 degrees of freedom, controlled by two actuators: the second and third phalanx are controlled by one actuator and coupled together. However, only the actuator controlling the first joint can apply a force in the direction of the opening of the finger, the other actuator can only bend the finger, not bring it back $^{2}$. This constrains us to use only the first actuator of each finger to apply a pressure on the outside of the finger. For this experiment, we also tie the second phalanx to the first one in order to rigidify the finger.

2) Procedure for experiment a: The goal of this experiment is to demonstrate the use of tactile sensors on the dorsal part of a robot for obstacle detection. The procedure is simple: the robot hand moves towards a flat surface (the obstacle) in a constant velocity Cartesian motion with the back of the fingers facing the obstacle. When contact is detected (the sensor value is above a threshold) with either finger (index or middle finger in that case), the motion stops to prevent collision and the hand is pulled back. We performed the obstacle detection experiment 20 times (see Fig. 7), with the contact occurring either on the proximal or the distal knuckle $^{3}$. The capacitance (proportional to pressure) of the sensor during the experiment is displayed in Fig 9.

3) Procedure for experiment $b$ : The experiment proceeds as follows: the robot positions its hand with its back towards a flat surface and while the hand is moving parallel to the plane, the fingers follow the contour of the surface, controlled in a pressure loop with the tactile sensors. The hand motion is a fixed linear Cartesian motion with constant velocity, while the fingers are controlled in current in order to maintain a desired tactile response.

A PD controller is implemented to follow a constant target pressure $\hat{s}_{f}$. This pressure is manually tuned so as to keep the fingers in contact without applying too

\footnotetext{
${ }^{1}$ The two other fingers are coupled and they cannot apply a force towards the back of the fingers because they are controlled by only one tendon. A spring brings back the joints to a straight position.

${ }^{2}$ Similarly, springs bring back the joints to a straight position when the tendon for bending is released.

${ }^{3}$ Between the $1^{\text {st }}$ and $2^{\text {nd }}$, or $2^{\text {nd }}$ and $3^{\text {rd }}$ joints. The knuckles can be seen on the index in the bottom of Fig. $6 b$.
} 


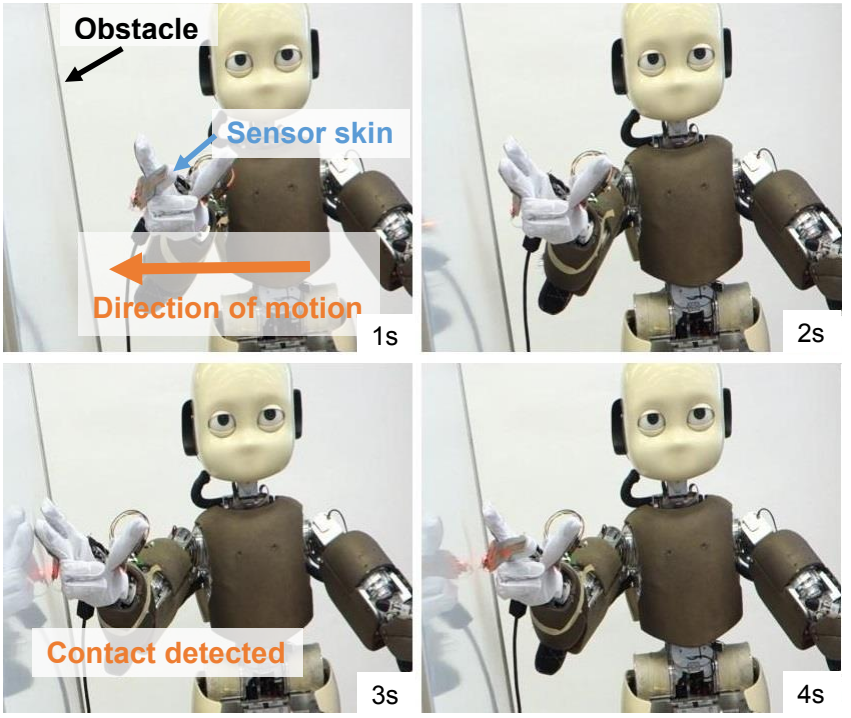

Fig. 7: Experiment 1: Snapshots from the obstacle detection task (distal knuckle). The hand moves towards the obstacle until contact is detected by the tactile sensors on the back of the fingers (here, second knuckle), then it withdraws.

much force on the object and finger tendons. Each finger $f$ is controlled in current $u_{f}$ following:

$$
u_{f}\left(s_{f}, \hat{s}_{f}\right)=\kappa_{p}\left(\hat{s}_{f}-s_{f}\right)-\kappa_{d} \dot{s}_{f}
$$

where $\dot{s}_{f}$ is the derivative of the total pressure at each finger, and $\kappa_{p} \in \Re$ and $\kappa_{d} \in \Re$ are the proportional and derivative coefficients ${ }^{4}$.

The two features can be seen on Fig. 8 and 10b: the arm and hand move parallel to the plane and the index follows the contours of the surface, including the features.

\section{B. Results}

1) Experiment a: The experiment is successful if the robot detects the contact and stops; it fails if the hand tries to force into the obstacle and must be stopped manually.

The experiment succeeded 20 out of 20 times for the second knuckle, only 17 out of 20 for the proximal knuckle (see Table I). The reason for the 3 failures is the lack of precision on the orientation of the hand: contact occurs on a part of the hand that is not covered with tactile skin and thus cannot be detected. This stresses the need for a tactile skin that covers all of the robot's surface. A noticeable delay of the robot reaction in the included video is a result of the robot control, as opposed to an insensitivity of the sensor.

\footnotetext{
${ }^{4}$ In this implementation, the gains $\kappa_{p}$ and $\kappa_{d}$ are hand-tuned.
}

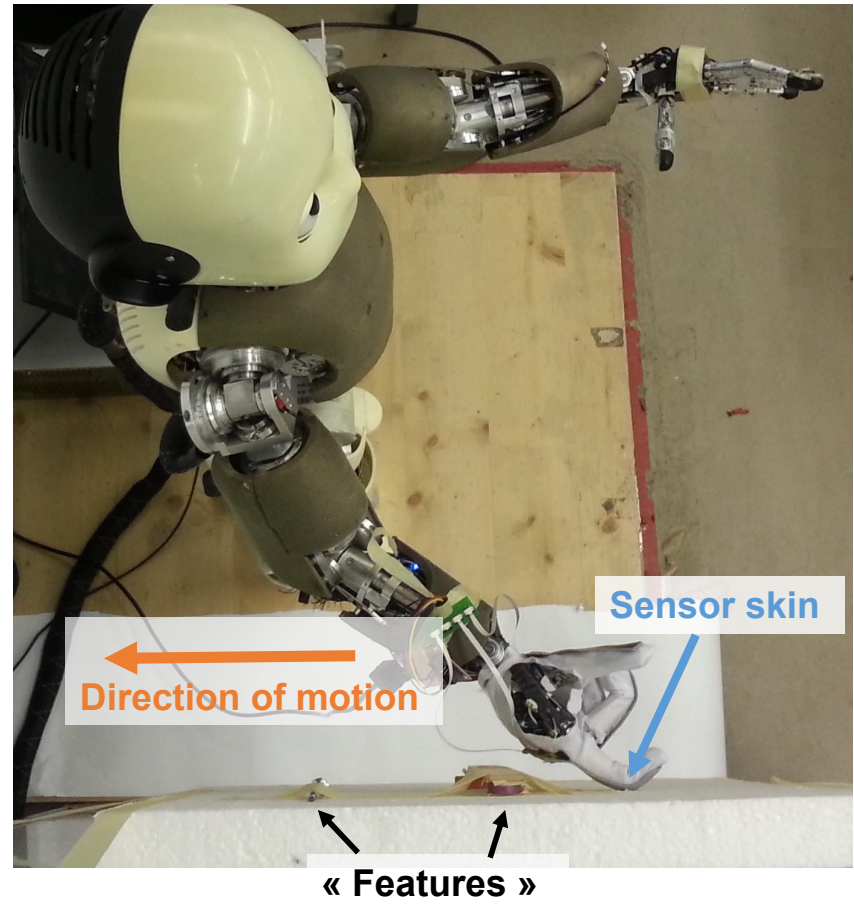

Fig. 8: Experiment 2: the index follows the contour of the features on the surface. Two "features" are present: a small and a bigger bump.

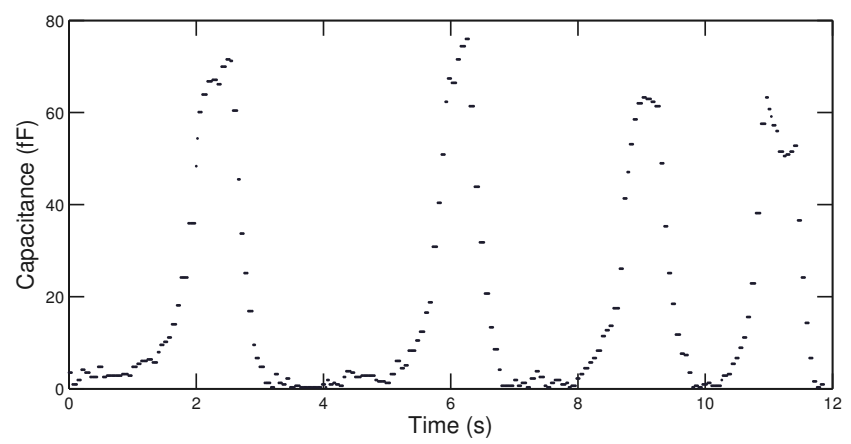

Fig. 9: The evolution of the capacitance value from the sensor that enters in contact during the obstacle detection experiment. The experiment is run 4 times in a row.

2) Experiment b: The two features are clearly extracted by the movement of the fingers thanks to the tactile sensors, as can be seen on Fig. 10. The lack of a perfect straight line between the two features can be attributed to the imprecision in the proprioceptive measurements of the robot that are used in the forward kinematics to compute the position of contact. Also, the precision of the reconstructed feature is limited by the length of the sensor $(1 \mathrm{~cm})$, which is the reason for the larger reconstructed feature around $200 \mathrm{~mm}$ on Fig. 


\begin{tabular}{c||c|c}
\hline Location of contact & \# trials & \# success \\
\hline Proximal knuckle & 20 & 17 \\
\hline Distal knuckle & 20 & 20 \\
\hline
\end{tabular}

TABLE I: Results of the obstacle detection, experiment a.

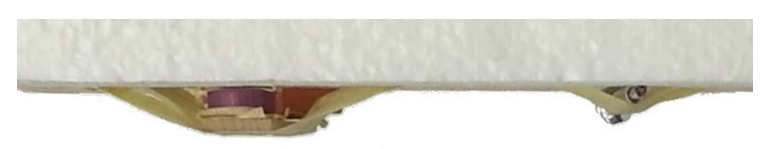

(a) Picture of the features

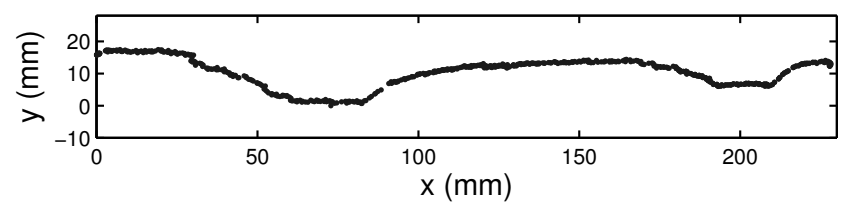

(b) Reconstructed features

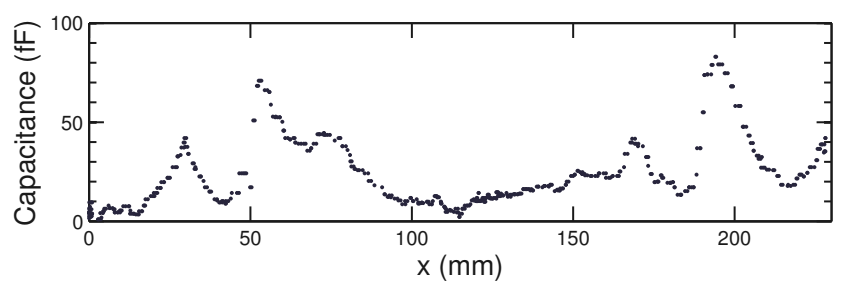

(c) Response of the sensor in contact during the feature following task

Fig. 10: a) A top-down picture of the two features. b) The reconstruction of the surface and features from 578 tactile data points collected with the artificial skin. c) The sensor's response during the scanning: the response increases when the finger enters in contact with the feature, and decreases when the finger releases the applied force.

$10 \mathrm{~b}$ compared to the true feature.

\section{CONCLUSIONS AND FUTURE WORK}

Stretchable tactile pressure sensors with integrated electronics have been mounted on a robotic hand and used to perform tactile exploration for the first time. The sensors, which can be subjected to tensile strains of $15 \%$ across a pressure range of $250 \mathrm{kPa}$, were mounted on a textile glove and worn by the iCub while performing the exploration. Results of obstacle detection and surface reconstruction tasks demonstrate the efficacy of and necessity for stretchable sensors for tactile sensing.

Further work includes an extensive electromehcanical characterization of the sensors under stretchable and/or compressive loading. Strategies to decouple the stretchable metallization sensitivity to strain from the capacitive sensor response will be explored. This decoupling will enable more complex tasks that extend those demonstrated in this paper such as three-dimensional reconstruction of surfaces. Ultimately it is important to understand both the influence of the robot on the sensors, including added noise from motors and cyclic strain on the materials, but also the influence of the sensors on the robot, including any negative impact such as an increase in the required torque for bending the fingers. The promise of the approach demonstrated in this work is the high sensitivity over a large dynamic range, both of which are not possible with existing resistive stretchable sensing solutions.

\section{ACKNOWLEDGMENT}

This research was supported by the Swiss National Science Foundation through the National Centre of Competence in Research (NCCR) Robotics and the ERC Starting Grant ESKIN (259419).

\section{REFERENCES}

[1] M. H. Lee, "Tactile sensing: new directions, new challenges," The International Journal of Robotics Research, vol. 19, no. 7, pp. 636-643, 2000.

[2] G. Schwartz, B. C.-K. Tee, J. Mei, A. L. Appleton, D. H. Kim, H. Wang, and Z. Bao, "Flexible polymer transistors with high pressure sensitivity for application in electronic skin and health monitoring," Nature Communications, vol. 4, p. 1859, 2013.

[3] L. Pan, A. Chortos, G. Yu, Y. Wang, S. Isaacson, R. Allen, Y. Shi, R. Dauskardt, and Z. Bao, "An ultra-sensitive resistive pressure sensor based on hollow-sphere microstructure induced elasticity in conducting polymer film," Nature Communications, vol. 5, 2014.

[4] P. Maiolino, M. Maggiali, G. Cannata, G. Metta, and L. Natale, "A flexible and robust large scale capacitive tactile system for robots," Sensors Journal, IEEE, vol. 13, no. 10, pp. 3910 3917, 2013.

[5] M. Ramuz, B. C.-K. Tee, J. B.-H. Tok, and Z. Bao, "Transparent, optical, pressure-sensitive artificial skin for large-area stretchable electronics," Advanced Materials, vol. 24, no. 24, pp. 3223-3227, 2012.

[6] C. Dagdeviren, Y. Su, P. Joe, R. Yona, Y. Liu, Y.-S. Kim, y. Huang, A. R. Damadoran, J. Xia, L. W. Martin, Y. Huang, and J. A. Rogers, "Conformable amplified lead zirconate titanate sensors with enhanced piezoelectric response for cutaneous pressure monitoring," Nature Communications, vol. 5, 2014.

[7] D. J. Lipomi, M. Vosgueritchian, B. C.-K. Tee, S. L. Hellstrom, J. A. Lee, C. H. Fox, and Z. Bao, "Skin-like pressure and strain sensors based on transparent elastic films of carbon nanotubes," Nature Nanotechnology, vol. 6, no. 12, pp. 788792, 2011. 
[8] F. L. Hammond III, Y. Menguc, and R. J. Wood, "Toward a modular soft sensor-embedded glove for human hand motion and tactile pressure measurement," in IEEE/RSJ International Conference on Intelligent Robots and Systems, Sep. 2014, pp. 4000-4007.

[9] Y.-L. Park, C. Majidi, R. Kramer, P. Berard, and R. J. Wood, "Hyperelastic pressure sensing with a liquid-embedded elastomer," Journal of Micromechanics and Microengineering, vol. 20, no. 12, p. 125029, 2010.

[10] N. Stubler, J. Fritzsche, and M. Kluppel, "Mechanical and electrical analysis of carbon black networking in elastomers under strain," Polymer Engineering \& Science, vol. 51, no. 6, pp. 1206-1217, 2011.

[11] M. Ying, A. P. Bonifas, N. Lu, Y. Su, R. Li, H. Cheng, A. Ameen, Y. Huang, and J. A. Rogers, "Silicon nanomembranes for fingertip electronics," Nanotechnology, vol. 23, no. 34, p. 344004, 2012.

[12] G. Buscher, R. Koiva, C. Schurmann, R. Haschke, and H. J. Ritter, "Tactile dataglove with fabric-based sensors," in $\mathrm{Hu}$ manoid Robots (Humanoids), 2012 12th IEEE-RAS International Conference on. IEEE, 2012, pp. 204-209.

[13] "Capacitive tactile sensing." [Online]. Available: http://www.pressureprofile.com/capacitive-sensors/
[14] N. Sommer and A. Billard, "Face classification using touch with a humanoid robot hand," in Humanoid Robots (Humanoids), 2012 12th IEEE-RAS International Conference on. IEEE, 2012, pp. 120-125.

[15] N. Sommer, M. Li, and A. Billard, "Bimanual compliant tactile exploration for grasping unknown objects," in IEEE International Conference on Robotics and Automation (ICRA), 2014.

[16] H. Vandeparre, D. Watson, and S. P. Lacour, "Extremely robust and conformable capacitive pressure sensors based on flexible polyurethane foams and stretchable metallization," Applied Physics Letters, vol. 103, no. 20, p. 204103, 2013.

[17] S. P. Lacour, S. Wagner, Z. Huang, and Z. Suo, "Stretchable gold conductors on elastomeric substrates," Applied Physics Letters, vol. 82, no. 15, pp. 2404-2406, 2003.

[18] T. Starner, "Human-powered wearable computing," IBM systems Journal, vol. 35, no. 3.4, pp. 618-629, 1996.

[19] S. A. Mascaro and H. H. Asada, "Photoplethysmograph fingernail sensors for measuring finger forces without haptic obstruction," Robotics and Automation, IEEE Transactions on, vol. 17, no. 5, pp. 698-708, 2001. 